\title{
Relação da Força Explosiva e Potência \\ Muscular com a Capacidade Funcional \\ no Processo de Envelhecimento
}

\author{
Correlation Between Explosive Strength and \\ Muscular Power With Funcional Capacity in the Aging Process
}

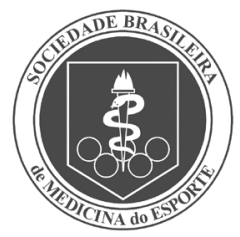

Artigo Original

Rodrigo Maciel Andrade

Sandra Marcela Mahecha Matsudo ${ }^{1}$

1. Centro de Estudos do Laboratório

de Aptidão Física de São Caetano do

Sul - Celafiscs

São Caetano do Sul São Paulo - SP

Endereço para correspondência:

rodmaciel@usp.br

sandra@celafiscs.org.br

Caixa Postal 168 CEP 09520-300

\begin{abstract}
RESUMO
Introdução: O declínio na força explosiva e potência muscular de membros inferiores (MMII) tem sido relacionado ao prejuízo funcional de idoso; entretanto, a influência do envelhecimento nestas variáveis, considerando os movimentos multiarticulares, não tem sido bem documentada. Objetivos: 1) comparar a força explosiva e a potência muscular de MMII de mulheres entre 50 e 79 anos em relação aos valores de referência aos 18 anos; e 2) associar essas variáveis com a capacidade funcional. Métodos: Foram avaliadas 227 mulheres não sedentárias, subdivididas nos grupos 50-59, 60-69 e 70-79 anos de idade. Como força explosiva foi considerada a impulsão vertical sem auxílio dos braços (FE) e como capacidade funcional a velocidade normal de andar (VEL), o tempo para levantar da cadeira (CAD) e o equilíbrio estático (EQ). A potência muscular (POT) foi estimada pela altura obtida no salto vertical. O valor correspondente aos 18 anos foi considerado como referência. Resultados: Foram observados menores valores $(p<0,05)$ em FE e POT nos três grupos de idade comparados aos 18 anos, sendo tais variáveis ainda menores no grupo 70-79 anos $(p<0,05)$. Somente o grupo $70-79$ anos apresentou menor valor $(p<0,05)$ em VEL e EQ. A alteração em FE comparada à POT foi significativamente maior em todos os grupos ( $p<0,05)$. À exceção da variável CAD nos grupos de 50-59 e 70-79 anos, a FE apresentou maior associação com a capacidade funcional do que a POT. Conclusão: FE e POT apresentaram menor valor quando comparadas ao grupo de 18 anos; entretanto, a POT não se altera em função da faixa etária. Contudo, a FE apresentou maior associação com a capacidade funcional.
\end{abstract}

Palavras-chave: velocidade normal de andar, levantar da cadeira, equilíbrio.

\begin{abstract}
Introduction: decrease in explosive strength and muscular power of lower limbs (LL) has been related to functional loss in the elderly; however, the influence of aging on these variables considering multiarticular movements are not well reported. Purpose: 1) to compare the explosive strength and power of LL in women between 50 and 79 years old, having the 18 year-old values as reference; 2 ) to study the relationship between these variables with functional capacity. Methods: 227 non-sedentary women were evaluated and divided into 3 groups: 50-59, 60-69 and 70-79 years old. The height of countermovement jump without movement arms (ES) was used as explosive strength index and normal walking velocity (VEL), time to stand from a chair (CHA) and static balance (SB) were used for functional capacity. Power (PO) was estimated by the jump height. The 18 year-old values were used as reference. Results: lower values $(p<0.05)$ in ES and PO were observed in the three groups when compared to reference, being those variables lower in 70-79 years $(p<0.05)$. Only 70-79 years group showed lower value $(p<0.05)$ in VEL and SB. Compared to PO, the change in ES was significantly higher in all groups $(p<0.05)$. Except for CHA in $50-59$ and $70-79$ years old groups, the ES showed higher association with the functional capacity than PO. Conclusion: The ES and PO showed changes when compared with the 18 year-old values, but PO did not change between the groups. However, ES showed higher correlation with functional capacity.
\end{abstract}

Keyswords: normal walking velocity, stand from a chair, balance.

\section{INTRODUÇÃO}

A potência muscular tem sido apontada como a melhor preditora de limitação funcional e declínio da qualidade de vida quando comparada à força máxima ${ }^{(1-5)}$, por representar o trabalho muscular por unidade de tempo, o que poderia ajudar na adoção de uma estratégia mais adequada para a execução de atividades diárias, como estabilização do corpo, prevenindo e/ou diminuindo o risco de queda ${ }^{(2)}$. Exemplos disto são os valores de correlações significantes encontrados na literatura entre potência muscular e capacidade funcional em idosos ${ }^{(1,3,6)}$

Outro ponto que tem recebido atenção durante o processo de envelhecimento é o comportamento das variáveis determinantes da potência muscular, força muscular e velocidade de deslocamento.

De Vito et al. ${ }^{(7)}$, ao analisarem estas variáveis, identificaram que as alterações na potência muscular decorrentes do avanço da idade poderiam ser melhor explicadas pela diminuição na velocidade de contração muscular do que pela capacidade de gerar força muscular. 
Corroborando com tais achados, uma diminuição significante na frequência de desenvolvimento de força ou força explosiva ${ }^{(8)}$, com o avanço da idade, tem sido relatada na literatura ${ }^{(9-13)}$, sugerindo que a velocidade para se atingir uma dada força parece ser mais importante do que a própria magnitude de força e potência para a manutenção da independência com o avanço da idade ${ }^{(2)}$.

Entretanto, os estudos que buscaram estabelecer respostas adaptativas destas variáveis no envelhecimento utilizaram mecanismos isotônicos ${ }^{(3)}$ e isocinéticos ${ }^{(4)}$ em sua determinação, portanto, com variação da sobrecarga, condição que não corresponde ao experimentado em ações diárias em que se faz necessário acelerar a própria massa corpórea, condição denominada isoinercial ${ }^{(14)}$.

Sendo assim, pode-se supor que a potência muscular e a força explosiva produzidas em condição isoinercial seriam capazes de representar melhor as adaptações na capacidade funcional experimentadas com o envelhecimento, também classificadas como isoinercial por dependerem da movimentação da massa corporal|(14).

Contudo, até o presente momento, não é de nosso conhecimento a existência de estudos que buscaram tal elucidação.

\section{OBJETIVOS}

Os objetivos do presente estudo foram: 1) comparar os indicadores de força explosiva e potência muscular de membros inferiores em muIheres de 50 a 59 anos, 60 a 69 anos e de 70 a 79 anos em relação aos valores de referência dos 18 anos de idade; e 2) associar os indicadores de força explosiva e potência muscular de membros inferiores com a capacidade funcional.

\section{MÉTODOS}

\section{Amostra}

A amostra, por conveniência do presente estudo, foi composta por 227 mulheres de 50 a 79 anos de idade (65 \pm 6 anos) que foram subdivididas em três grupos de acordo com a idade cronológica: 50-59 anos $(n=39), 60-69$ anos $(n=127)$ e $70-79$ anos $(n=58)$. Todas as mulheres frequentavam um centro social e recreacional para a terceira idade, onde o Centro de Estudos do Laboratório de Aptidão Física de São Caetano do Sul (Celafiscs) realiza, desde 1997, o Projeto Longitudinal de Envelhecimento e Aptidão Física de São Caetano do Sul.

Os critérios de inclusão adotados foram: fazer parte do programa de exercícios físicos oferecidos pelo centro há pelo menos seis meses e ter frequência nas aulas igual ou superior a 75\%, enquanto os critérios de exclusão foram: presença de problemas de locomoção e cognição, ser funcionalmente dependente e não ter realizado todos os testes funcionais. Todas as participantes assinaram um termo de consentimento livre e esclarecido, previamente aprovado pelo comitê de ética em pesquisa da Universidade Federal de São Paulo - Escola Paulista de Medicina.

As aulas foram realizadas duas vezes por semana em dias alternados (terças e quintas-feiras ou quartas e sextas-feiras), no período matutino, com duração de 50 minutos por aula, com grupo de aproximadamente
30 senhoras por turma. As atividades realizadas foram subdivididas em atividades de aquecimento, atividades aeróbicas, de relaxamento e alongamento e exercícios de fortalecimento muscular.

Durante as aulas, a frequência cardíaca média foi de 97,3 $\pm 13,8 \mathrm{bpm}$ $(73,9 \pm 12,1$ a 131,4 $\pm 26,4)$, representando aproximadamente $68 \%$ da frequência cardíaca máxima prevista(15). A avaliação do nível de atividade física utilizando o questionário internacional de atividade física (IPAQ) realizada nas participantes do projeto tem mostrado que $71,1 \%$ delas são classificadas como suficientemente ativas e 28,9\% como insuficientemente ativas ${ }^{(16)}$.

Foram medidas a massa corporal e estatura, e calculado o índice de massa corporal (IMC). Como indicador da força explosiva foi considerada a altura de salto vertical sem auxílio dos braços (FE)(18) a potência muscular isoinercial (POT) foi estimada a partir da altura de salto ${ }^{(17)} \mathrm{e}$ normalizadas pela massa corporal.

Como indicador da capacidade funcional, foi considerada a velocidade normal de andar (VEL), tempo para levantar da cadeira (CAD) e equilíbrio estático (EQ) ${ }^{(18)}$.

A aplicação dos testes se deu de forma aleatória sistemática, em três tentativas, com intervalos de um minuto entre cada tentativa e de 15 minutos entre cada teste. Foram considerados para análise a maior altura de salto (FE), a maior velocidade média - estimada a partir do tempo gasto para percorrer a distância do teste (VEL) -, o menor intervalo de tempo para levantar da cadeira (CAD) e o maior intervalo de tempo em equilíbrio unipodal (EQ).

Para o estudo da FE e POT, de acordo com a faixa etária, foi utilizado como referência (100\%) para o presente estudo o valor respectivo aos 18 anos de idade, obtido a partir do valor normativo populacional utilizado para diagnóstico, prescrição e prognóstico de aptidão física geral ${ }^{(19)}$.

\section{ANÁLISE ESTATÍSTICA}

Para determinação da evolução, de acordo com a faixa etária nas variáveis FE e POT, foram utilizados o delta percentual ( $\triangle \%$ ) entre o valor obtido para cada faixa etária e o valor correspondente aos 18 anos, e aproximação para curva normal reduzida para comparativo destes valores. Para as comparações entre as faixas etárias foi utilizada a análise de Kruskall-Wallis e post hoc de Tukey, quando necessário. A correlação de Spearman Rho foi adotada para associar a força explosiva e a potência muscular com a capacidade funcional, uma vez que os dados não apresentaram distribuição normal, segundo Kolmogorov-Smirnov. O nível de significância adotado para todas as análises foi de $p<0,05$.

\section{RESULTADOS}

Na tabela 1 são apresentados dados de caracterização da amostra estudada, não sendo encontrada diferença estatisticamente significante ( $p<0,05)$ entre os grupos para estas variáveis.

A força explosiva e a potência muscular em todos os grupos apresentaram valores significativamente menores $(p<0,05)$ quando comparados aos valores de 18 anos, sendo que o valor encontrado no grupo de 70-79 anos foi ainda menor $(p<0,05)$ se considerados os outros grupos, como demonstrado no gráfico 1.

Tabela 1. Valores em média ( $\bar{x}$ ) e desvio padrão (ఠ) das variáveis: idade, massa corporal, estatura e IMC dos grupos de acordo com a idade cronológica.

\begin{tabular}{c|c|c|c|c|c|c|c|c}
\hline Faixa etária & \multicolumn{2}{|c|}{ Idade (anos) } & \multicolumn{2}{c|}{ Massa corporal (kg) } & \multicolumn{2}{c}{ Estatura (cm) } & \multicolumn{1}{c}{ IMC (kg/m $)$} \\
\hline & $\bar{x}$ & $\boldsymbol{\sigma}$ & $\bar{x}$ & $\boldsymbol{\sigma}$ & $\bar{x}$ & $\boldsymbol{\sigma}$ & $\overline{\boldsymbol{\sigma}}$ \\
\hline $50-59$ & 55,79 & 2,65 & 69,76 & 13,18 & 156,43 & 5,50 & 28,59 & 4,89 \\
\hline $60-69$ & 64,75 & 2,90 & 67,65 & 10,65 & 156,42 & 6,20 & 27,79 & 3,73 \\
\hline $70-79$ & 72,62 & 2,53 & 67,04 & 10,10 & 155,21 & 6,53 & 28,03 & 4,27 \\
\hline
\end{tabular}




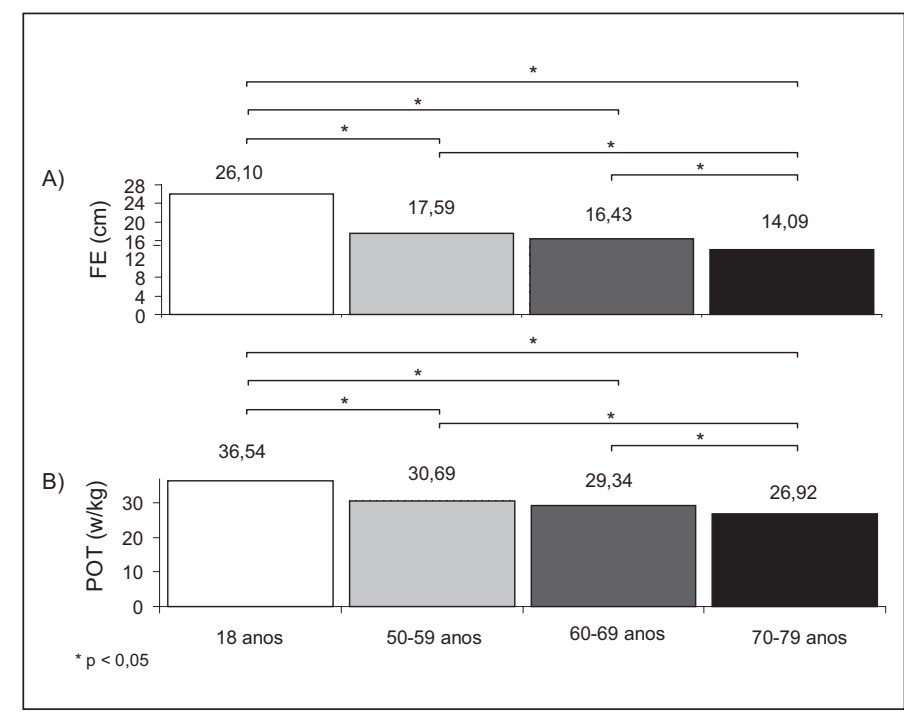

Gráfico 1. Valores dos indicadores da força explosiva - FE (A) e potência muscular POT (B) nos grupos de acordo com a idade cronológica.

Somente o grupo 70-79 anos apresentou menor valor $(p<0,05)$ da velocidade de andar (VEL) e o equilíbrio estático (EQ) frente aos valores de 18 anos (gráfico 2).

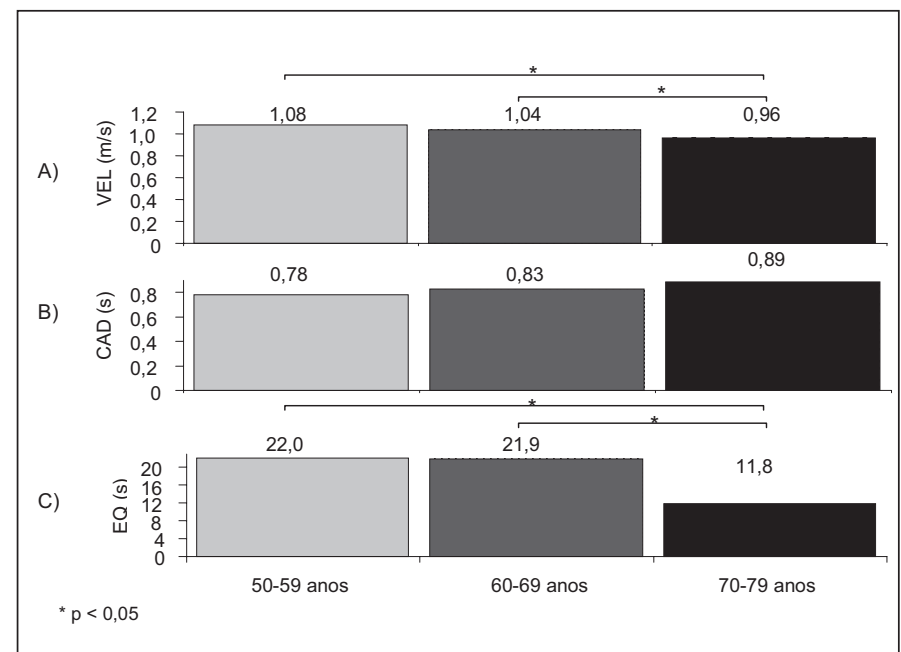

Gráfico 2. Valores de velocidade normal de andar - VEL (A), tempo para levantar da cadeira - CAD (B) e equilíbrio estático - EQ (C) nos grupos de acordo com a idade cronológica.

No gráfico 3 são apresentados os deltas percentuais para força explosiva (FE) e potência muscular (POT) de cada grupo comparado ao valor aos 18 anos de idade, sendo que o grupo 70-79 anos apresentou valor de maior magnitude $(p<0,05)$ em ambas variáveis.

$\mathrm{Na}$ análise comparativa entre os deltas percentuais de FE e POT de cada grupo, maior valor $(p<0,05)$ foi observado em FE em todos os grupos (gráfico 4).

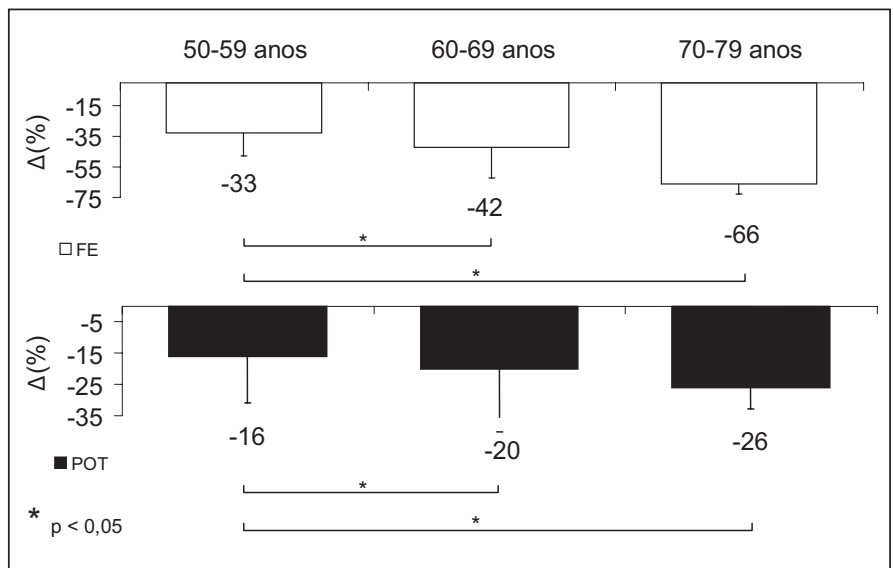

Gráfico 3. Delta percentual do indicador de força explosiva (FE) e potência muscular (POT) em relação ao valor de referência de acordo com a idade cronológica.

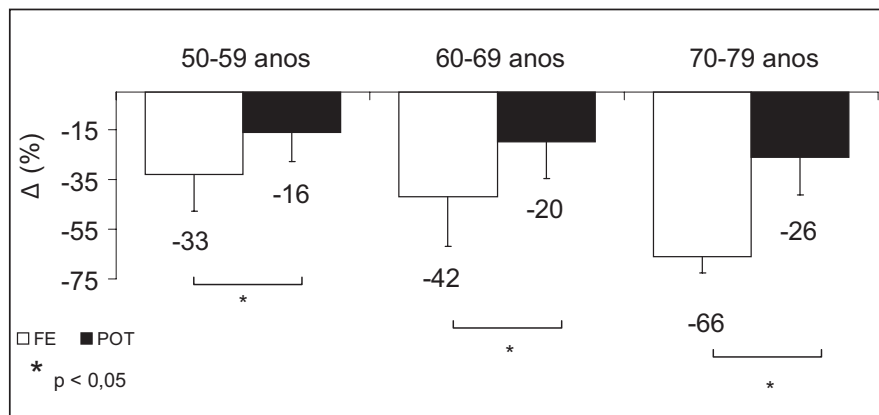

Gráfico 4. Valores comparativos dos deltas percentuais de FE e POT nos grupos de acordo com a idade cronológica.

À exceção da variável levantar da cadeira no grupo 50-59 anos, a FE apresentou melhor associação com a capacidade funcional do que a POT, sendo tal associação significante $(p<0,05)$, principalmente no grupo 60-69 anos.

\section{DISCUSSÃO}

O salto vertical tem sido amplamente utilizado em todo o mundo na investigação de práticas desportivas, modelos de treinamento, além de ser uma variável importante na determinação da aptidão física. Dentre os diferentes recursos possíveis para a quantificação dos valores de salto, a determinação da força de reação do solo (FRS) tem sido proposta como método padrão(20), tendo sua fundamentação por princípios físicos ${ }^{(21)}$, que utilizaremos de forma sucinta apenas para justificar a altura de salto vertical como indicativo da força explosiva.

De forma inicial, torna-se inevitável à apresentação do princípio quantidade de movimento linear $(\mathrm{Q})$, uma grandeza que relaciona massa (m) e velocidade de um corpo (v), que matematicamente pode ser expressa como ${ }^{(21)}$ :

\section{$\mathrm{Q}=\mathrm{m}^{*} \mathrm{v} \quad$ Equação 1}

De forma específica ao salto vertical, é inquestionável a variação da força muscular aplicada durante as fases concêntrica e excêntrica;

Tabela 2. Coeficientes de correlação entre altura de salto (FE) e potência muscular (POT) com a velocidade normal de andar (VEL), tempo para levantar da cadeira (CAD) e equilíbrio estático (EQ) dos grupos acordo com a idade cronológica.

\begin{tabular}{c|c|c|c|c|c|c}
\hline & \multicolumn{2}{|c|}{$50-59$ anos } & \multicolumn{2}{c}{$\mathbf{6 0 - 6 9}$ anos } & \multicolumn{2}{c}{ 70-79 anos } \\
\cline { 2 - 7 } & FE & POT & FE & POT & PE & $-0,03$ \\
\hline $\operatorname{VEL}(\mathrm{m} / \mathrm{s})$ & 0,12 & $-0,03$ & $0,24^{*}$ & 0,15 & 0,12 \\
\hline CAD (s) & 0,01 & 0,11 & $-0,27^{*}$ & $-0,22^{*}$ & $0,37^{*}$ & $0,37^{*}$ \\
\hline EQ (s) & 0,19 & 0,10 & $0,26^{*}$ & 0,14 & 0,08 \\
\hline
\end{tabular}


sendo assim, a partir da grandeza física impulso (I), é definido como a variação da quantidade de movimento linear $(\Delta Q)$ pode-se quantificar o movimento.

$$
I=\Delta \mathrm{Q}
$$$$
\text { I = Qfinal - Qinicial }
$$

Equação 2

Por substituição da Equação 1, impulso é matematicamente definido como:

$$
I=(\text { mfinal * vfinal })-(\text { minicial * vinicial }) \quad \text { Equação } 3
$$

Visto que a massa corporal $(m)$ é constante, o impulso é:

$$
\begin{array}{ll}
\mathrm{I}=\mathrm{m} *(\text { vfinal }- \text { vinicial }) & \text { Equação } 4 \\
\mathrm{I}=\mathrm{m}^{*} \Delta \mathrm{v} & \text { Equação } 5
\end{array}
$$

Tendo ainda a segunda lei de movimento que diz que a taxa de variação de quantidade de movimento linear é proporcional à força aplicada, e na direção em que a força age, esta pode ser apresentada como:

$$
\mathrm{F}=\mathrm{m}^{*} \mathrm{a} \quad \text { Equação } 6
$$

Sendo $\mathrm{F}$ a força aplicada, $\mathrm{m}$ a massa corporal e a variação da velocidade $(\Delta v)$ no tempo $(\Delta t)$ ou aceleração. Logo, por substituição:

$$
\begin{array}{lc}
F=m^{*}(\Delta v / \Delta t) & \text { Equação } 7 \\
\Delta t{ }^{*} F=m^{*} \Delta v & \\
\text { Como apresentado na Equação 5, impulso é expresso como: } \\
I=m^{*} \Delta v & \text { Equação } 5 \\
\text { Logo: } & \text { Equação } 8
\end{array}
$$

Desta forma, a altura de salto vertical é o resultado da magnitude de força gerada por unidade de tempo, também apresentada como força explosiva ${ }^{(8)}$.

Contudo, a força explosiva tem recebido atenção mais recentemente, sendo que anterior a tal foco de investigação, a potência muscular era apresentada como o melhor indicativo de funcionalidade e performance, o que fez com que Sayers et al. ${ }^{(17)}$ propusessem uma equação válida para a determinação da potência muscular a partir da altura de salto, sendo, portanto, um método de fácil acesso e baixo custo.

Quanto aos resultados encontrados neste estudo, as diferenças não significantes $(p<0,05)$ da massa corporal, estatura e IMC entre as faixas etárias, que ratificam o estudo de De Vito et al. ${ }^{(7)}$ que reportaram mudança não significativa nas variáveis antropométricas e de adiposidade em mulheres de 50 a 75 anos.

Como apresentado no gráfico 1, a FE e POT nos grupos 50-59 e 60-69 anos apresentaram valores significativamente menores $(p<0,05)$ aos reportados aos 18 anos de idade (-33\% e -16\%; -42\% e -22\%) com diferenças ainda maiores $(p<0,05)$ quando considerado o grupo de 70-79 anos (-66\% e -26\%). Estes valores corroboram com Izquierdo et al. ${ }^{(13)}$ que encontraram valor 29,1\% menor nos indivíduos fisicamente ativos entre 65 a 74 anos quando comparado a indivíduos com as mesmas características entre 18 e 30 anos; e com Andrade et al. ${ }^{(22)}$ que, utilizando método semelhante ao adotado no presente estudo, encontraram valor significantemente menor $(p<0,05)$ na altura de salto (FE) em 33,8\%, 46,9\% e 53,2\% nas faixas etárias 50-59, 60-69 e 70-79 anos, respectivamente.

Os valores de POT apresentados no atual estudo corroboram com Ferretti et al. ${ }^{(23)}$, que identificaram valor 19,3\% menor na potência muscular obtida no salto sem contramovimento ao comparar indivíduos sedentários entre 20 e 35 anos e indivíduos com mais de 50 anos, porém, não sendo esta diferença significante $(p<0,05)$.

Isso vai de encontro ao reportado por Skelton et al. ${ }^{(6)}$ que apresentaram diminuição aproximada de 35\% na POT por década de vida e com Macaluso e De Vito ${ }^{(4)}$ e Runge et al. ${ }^{(5)}$, que reportaram valores de potência muscular no leg press $60,9 \%$ menores e significantes $(p<0,05)$ em mulheres ativas entre 65 e 74 anos quando comparadas às mulheres ativas entre 18 e 30 anos; e valor 50\% menor e significante $(p<0,05)$ ao analisar a potência muscular a partir do salto com contramovimento em homens de 20 a 88 anos e mulheres de 18 a 79 anos $^{(5)}$.

Como apresentado no gráfico 4, quando comparadas ao valor de referência, maiores diferenças $(p<0,05)$ em FE foram observadas frente à POT em todos os grupos; contudo, a inexistência de estudo com semelhante amostra e método de coleta de dados inviabiliza qualquer comparação com outros dados.

A associação encontrada entre FE e levantar da cadeira no grupo 70-79 anos de idade corrobora com Tanaka et al.(24) que reportaram coeficientes de correlação não significantes $(p<0,05)$ de $-0,11$. No grupo 60-69 anos foi encontrado maior magnitude de associação em nosso estudo $(-0,27$ versus $-0,03)$.

Quando considerados a FE e velocidade normal de andar, os resultados encontrados apresentaram magnitudes condizentes a outros estudos ${ }^{(24)}$, porém em sentido inverso. Entretanto, cabe salientar que no estudo citado, os autores ${ }^{(24)}$ adotaram como índice de velocidade normal de andar o tempo necessário para percorrer a distância de 3,33 metros, enquanto que, em nosso estudo, foi calculada a velocidade média no transcorrer deste percurso.

As associações entre POT e equilíbrio estático nos grupos 50-59 e 60-69 anos estão de acordo com o estudo de Bean et al. ${ }^{(1)}$ que reportaram correlação não significante $(p<0,001)$ de 0,11 entre tais variáveis. Entretanto, no grupo 70-79 anos, as correlações encontradas no presente estudo apresentaram mesmo sentido, porém com maior magnitude se comparadas ao estudo de Bean et al.(1).

As associações entre POT e velocidade de andar apresentaram valores de baixa magnitude por vezes positivas, por vezes negativas, respostas não condizentes com o reportado por Bean et al. (1) que foram positivas e significantes ( $p<0,001$ ) entre 0,54 e 0,56.

Fenômeno semelhante ao reportado na associação da velocidade de andar e POT foi encontrado quando considerado o tempo de levantar da cadeira e a POT, sendo encontrado nos grupos 50-59 e 70-79 anos valores de baixa magnitude não significantes $(p<0,05)$. Contudo, no grupo 60-69 anos, o valor de correlação foi significante $(p<0,05)$ com sentido e magnitude $(-0,22)$ condizente à relatada por Bean et al. ${ }^{(1)}$, que foi de $-0,31$ e $-0,32$, ambas significantes ( $\left.p<0,001\right)$.

Acreditamos que as diferenças, significantemente maiores ( $p<$ 0,05), obtidas em FE ao serem confrontadas ao valor de referência, assim como as maiores magnitudes dos valores de correlação desta com as variáveis de capacidade funcional, possam melhor representar as alterações morfológicas e neurais experimentadas com o envelhecimento. A razão pela qual tal hipótese é considerada surge do fato de que a força explosiva esta associada a frequência de desenvolvimento de força ${ }^{(25-27)}$ variável, grandemente influenciada pelo avanço da idade ${ }^{(9)}$.

Klass et al. ${ }^{(9)}$ ao compararem indivíduos entre 18 e 22 anos e indivíduos entre 71 e 84 anos, relataram que o envelhecimento teve maior influência na frequência de desenvolvimento de torque do que na própria grandeza do torque máximo produzido, e seria consequência de diminuição na amplitude e frequência de disparo motor e maior período de latência neuronal, o que dificultaria alcançar o limiar de potencial de ação necessário para contração de maior número de unidades motoras.

Desta forma, tais achados corroboram com nossa hipótese da altura de salto (FE) ser considerada melhor indicativo das adaptações do envelhecimento, pois como evidenciado anteriormente, a altura de salto depende de quão rápido o indivíduo consegue desenvolver força muscular (25-27), variável amplamente prejudicada com o envelhecimento ${ }^{(9,28)}$. Deste modo, nossos resultados corroboram com De Vito et al. ${ }^{(7)}$ que propõem o teste de salto vertical como sendo um teste funcional capaz de refletir muitas 
atividades diárias realizadas com movimentos balísticos que necessitam de aceleração e desaceleração da massa corporal.

Acreditamos que os menores valores encontrados para força explosiva e potência frente aos observados aos 18 anos de idade sejam, provavelmente, consequência de fatores neurais $(7,29)$, que produziriam alterações na velocidade de contração muscular(4,7,9,11,12,23,28) assim como coordenação intra e intermuscular ${ }^{(7)}$.

Apesar da sarcopenia não ser capaz de explicar os menores valores de potência em outros estudos ${ }^{(7,23)}$, esta não deve ser descartada como fator que poderia influenciar os resultados observados no presente estudo, uma vez que possíveis mudanças no conteúdo de gordura interna muscular, nos tecidos conectivos e ângulo de peneação das fibras não puderam ser identificadas pelos métodos adotados. Ainda, visto que a massa corporal foi utilizada na equação para a determinação da potência muscular, esta pode ainda ter contribuído na análise da evolução de acordo com a faixa etária $(\Delta \%)$ da potência muscular.
Desta forma, acreditamos que as alterações encontradas na altura de salto possam ser utilizadas como indicativo de força explosiva, e que a altura de salto poderia representar as alterações morfológicas e neurais, características do envelhecimento e comprometimento funcional.

\section{CONCLUSÃO}

A força explosiva e a potência muscular foram significantemente menores nos sujeitos de 50 a 79 anos de idade quando comparado aos valores de 18 anos de idade, sendo que a potência muscular apresentou menor magnitude de alteração quando comparado à força explosiva. Entretanto, a força explosiva teve maior associação com a capacidade funcional, principalmente na faixa etária 60 e 69 anos.

Todos os autores declararam não haver qualquer potencial conflito de interesses referente a este artigo.

\section{REFERÊNCIAS}

1. Bean JF, Kiely DK, LaRose S, Alian J, Frontera WR, Is stair climb power a clinically relevant measure of leg power impairments in at-risk older adults? Arch Phys Med Rehabil 2007;88:604-9.

2. Sayers SP, High-speed power training: a novel approach to resistance training in older men and women. A brief review and pilot study. J Strength Cond Res 2007;21:518-26.

3. Capodaglio P, Capodaglio EM, Ferri A, Scaglioni G, Marchi A, Saibene F, Muscle function and functional ability improves more in community-dwelling older women with a mixed-strength training programme. Age Ageing 2005;34:141-7.

4. Macaluso A, De Vito G, Comparison between young and older women in explosive power output and its determinants during a single leg-press action after optimisation of load. Eur J Appl Physiol 2003:90:458-63.

5. Runge M, Rittweger J, Russo CR, Schiess I H, Felsenberg $D$, Is muscle power output a key factor in the age-related decline in physical performance? A comparison of muscle cross section, chair-rising test and jumping power. Clin Physiol Funct Imaging 2004;24:335-40.

6. Skelton DA, Greig CA, Davies JM, Young A, Strength, power and related functional ability of healthy people aged 65-89 years. Age Ageing 1994;23:371-7.

7. De Vito G, Bernardi M, Forte R, Pulejo C, Macaluso A, Figura F, Determinants of maximal instantaneous muscle power in women aged 50-75 years. Eur J Appl Physiol Occup Physiol 1998;78:59-64.

8. Carvalho C, Carvalho A, Não se deve identificar força explosiva com potência muscular, ainda que existam algumas relações entre ambas. Rev Port Cien Desp 2006;6:241-8.

9. Klass M, Baudry S, Duchateau J, Age-related decline in rate of torque development is accompanied by lower maximal motor unit discharge frequency during fast contractions. J Appl Physiol 2008;104:739-46.

10. Henwood TR, Riek S, Taaffe DR, Strength versus muscle power-specific resistance training in communitydwelling older adults. J Gerontol A Biol Sci Med Sci 2008;63:83-91.

11. Petrella JK, Kim JS, Tuggle SC, Hall SR, Bamman MM, Age differences in knee extension power, contractile velocity, and fatigability. J Appl Physiol 2005;98:211-20.

12. Lanza IR, Towse TF, Caldwell GE, Wigmore DM, Kent-Braun JA, Effects of age on human muscle torque, velocity, and power in two muscle groups. J Appl Physiol 2003;95:2361-9.

13. Izquierdo M, Ibanez J, Gorostiaga E, Garrues M, Zuniga A, Anton A, et al., Maximal strength and power characteristics in isometric and dynamic actions of the upper and lower extremities in middle-aged and older men. Acta Physiol Scand 1999;167:57-68.

14. Hopkins WG, Schabort EJ, Hawley JA, Reliability of power in physical performance tests. Sports Med 2001:31:211-34.
15. Matsudo SMM, Evolução da aptidão física e da capacidade funcional de mulheres ativas de 50 anos de idade de acordo com a idade cronológica. 2001. f. Doutorado (Departamento de Reabilitação) Universidade Federal de São Paulo, São Paulo.

16. Cruciani F, Araújo TLd, Matsudo SMM, Matsudo VKR, Nível de atividade física de mulheres acima de 50 anos participantes de programa de atividade física. In:50 Congresso Brasileiro de Atividade Física e Saúde. 2005;184.

17. Sayers SP, Harackiewicz DV, Harman EA, Frykman PN, Rosenstein MT, Cross-validation of three jump power equations. Med Sci Sports Exerc 1999;31:572-7.

18. Matsudo SMM, Avaliação da aptidão física. In: Matsudo SMM editor Avaliação do Idoso - Física e funcional. Londrina: Midiograf, 2005;23-62.

19. Matsudo VKR, Critérios biológicos para diagnóstico, prescrição e prognóstico de aptidão física em escolares de 7 a 18 anos de idade. 1991. 127 f. Livre docência (Ciências da Saúde) - Universidade Gama Filho, Rio de Janeiro.

20. Hatze $H$, Validity and Reliability of Methods for Testing Vertical Jumping Performance. J Appl Biomech 1998;14:127-40

21. Hall S, Biomecânica Básica. 4 ed. Rio de janeiro: Guanabara Koogan; 2005.

22. Andrade ELd, Matsudo SMM, Matsudo VKR, Performance neuromotora em mulheres ativas. Rev Bras Ativ Fis Saúde 1995;1:5-14.

23. Ferretti G, Narici MV, Binzoni T, Gariod L, Le Bas JF, Reutenauer H, et al. Determinants of peak muscle power: effects of age and physical conditioning. Eur J Appl Physiol Occup Physiol 1994;68:111-5.

24. Tanaka MS, Matsudo SMM, Matsudo VKR, Associação de variáveis neuromotoras da aptidão física e a capacidade funcional de idosas fisicamente ativas. In:XXVII Simpósio Internacional de Ciências do Esporte. 2004;51.

25. Kollias I, Hatzitaki V, Papaiakovou G, Giatsis G, Using principal components analysis to identify individual differences in vertical jump performance. Res Q Exerc Sport 2001;72:63-7.

26. Thorlund JB, Aagaard P, Madsen K, Rapid muscle force capacity changes after soccer match play. Int J Sports Med 2009;30:273-8.

27. Laffaye G, Bardy BG, Durey A, Principal component structure and sport-specific differences in the running one-leg vertical jump. Int J Sports Med 2007;28:420-5.

28. Toji H, Kaneko M, Effects of aging on force, velocity, and power in the elbow flexors of males. J Physiol Anthropol 2007;26:587-92

29. Ferretti G, Gussoni M, Di Prampero PE, Cerretelli P, Effects of exercise on maximal instantaneous muscular power of humans. J Appl Physiol 1987:62:2288-94. 